\title{
Evaluation of Morphological Changes in Degenerative Cartilage Using 3-D Optical Coherence Tomography
}

\author{
Jong-In Youn* \\ Department of Biomedical Engineering, College of Health and Medical Science, Catholic University \\ of Daegu, 712-702, Republic of Korea
}

(Received April 7, 2008 : revised April 15, 2008 : accepted April 16, 2008)

\begin{abstract}
Optical Coherence Tomography (OCT) is an important noninvasive medical imaging technique that can reveal subsurface structures of biological tissue. OCT has demonstrated a good correlation with histology in sufficient resolution to identify morphological changes in articular cartilage to differentiate normal through progressive stages of degenerative joint disease. Current OCT systems provide individual cross-sectional images that are representative of the tissue directly under the scanning beam, but they may not fully demonstrate the degree of degeneration occurring within a region of a joint surface. For a full understanding of the nature and degree of cartilage degeneration within a joint, multiple OCT images must be obtained and an overall assessment of the joint surmised from multiple individual images. This study presents frequency domain threedimensional (3-D) OCT imaging of degenerative joint cartilage extracted from bovine knees. The 3-D OCT imaging of articular cartilage enables the assembly of 126 individual, adjacent, rapid scanned OCT images into a full 3-D image representation of the tissue scanned, or these may be viewed in a progression of successive individual two-dimensional (2-D) OCT images arranged in 3 -D orientation. A fiber-based frequency domain OCT system that provides cross-sectional images was used to acquire 126 successive adjacent images for a sample volume of $6 \times 3.2 \times 2.5 \mathrm{~mm}^{3}$. The axial resolution was $8 \mu \mathrm{m}$ in air. The 3-D OCT was able to demonstrate surface topography and subsurface disruption of articular cartilage consistent with the gross image as well as with histological cross-sections of the specimen. The 3-D OCT volumetric imaging of articular cartilage provides an enhanced appreciation and better understanding of regional degenerative joint disease than may be realized by individual 2-D OCT sectional images.
\end{abstract}

Keyword: Articular cartilage, Degeneration, Morphology, Surface irregularity

OCIS codes : (170.0170) Medical optics and biotechnology; (170.1610) Clinical applications; (170.3880) Medical and biological imaging; (170.4500) Optical coherence tomography; (170.4580) Optical diagnostics for medicine

\section{INTRODUCTION}

Degenerative disease of the joints, also called osteoarthritis, is the most common joint disorder and is characterized by degeneration of articular cartilage [1]. Although patients may experience dysfunction or pain resulting from acute cartilage injury, they will eventually suffer from degenerative arthritis. Early signs of this cartilage degeneration do not affect the full thickness of the tissue and may include fibrillation, and flaps [1]. Non-destructive imaging methods like X-ray,

*Corresponding author: jyoun@cu.ac.kr computed tomography (CT), and ultrasound do not image soft tissue well and have relatively poor axial resolutions, which prevent identification of microstructural changes. Clinicians currently use these conventional imaging techniques or arthroscopy to obtain macroscopic information concerning articular cartilage degeneration. Currently, examination of tissue microarchitecture is performed by destructive mechanical biopsy with permanent removal of the relevant tissue for histology analysis. Mankin et al. classified six different stages for cartilage degeneration as seen in Figure 1 [2]. From Mankin 1 (M1) to Mankin 5 (M5) stages, cartilage shows 
surface irregularities and clefts to the different zones. Eventually, Makin 6 (M6) stage shows collagen matrix disorganization with permanent degeneration in cartilage [2].

Minimally invasive arthroscopic imaging with arthroscopy is considered the clinical standard for assessment of cartilage and the arthroscopy allows a magnified view in the defect area and gross surface smoothness. However, this arthroscopy does not allow cross-sectional imaging below the surface.

Optical Coherence Tomography (OCT) is a noninvasive imaging technology that uses light reflected from within a tissue to generate two dimensional, sectional images of the tissue structure in a manner similar to but with higher resolution than is obtained with ultrasound. OCT is reported to provide images that have good correlation with histology in sufficient resolution to identify morphological changes in articular cartilage that allows assessment of cartilage thickness up to 2.5 $\mathrm{mm}$ and differentiation between normal through progressive stages of degenerative joint disease [3-9]. Current OCT systems provide individual cross-sectional images that are representative of the tissue directly under the scanning beam; however, images of an individual section may not fully demonstrate the degree of degeneration or structural alteration that occurs within a region of a joint surface. For a full understanding of the nature and degree of cartilage degeneration within a joint, multiple OCT images must be obtained and an overall assessment of the joint surmised from evaluation of multiple individual images. A fiber-based OCT system that obtains sectional images with an axial resolution of $8 \mu \mathrm{m}$ was used to acquire 126 successive adjacent images of $6 \mathrm{~mm} \times 3.2 \mathrm{~mm} \times 2.5 \mathrm{~mm}$ specimens of bovine articular cartilage. The 3 -D OCT imaging procedure was used to assemble each set of 126 individual, adjacent, rapid-scanned frequency domain OCT images into a full 3-D image representation of the specimen, and these scans were also used to generate a progression of successive individual two-dimensional (2-D) OCT images selectively viewed in $\mathrm{x}, \mathrm{y}$ or $\mathrm{z}$ planes relative to the tissue surface. The 3-D OCT images demonstrate surface topography and subsurface disruption of articular cartilage consistent with the gross image as well as with histological cross-sections of the specimen.

\section{EXPERIMENTAL METHODS}

Fresh bovine cadaver knees were obtained from a local abattoir. The patella, distal femur, and proximal tibia were isolated following removal of supporting muscle, tendons, ligaments, and joint capsule. The distal femur and patella were individually wrapped in $0.9 \%$ saline soaked gauze sponges and cotton toweling, placed in sealed plastic bags and stored at $4{ }^{\circ} \mathrm{C}$ until used. Just prior to each experiment a specimen bag was removed from refrigeration and allowed to equilibrate to room temperature. Five samples were obtained from areas determined by visual examination of cartilage in various stages of degenerative joint disease as evidenced by partial to full thickness erosions and surface irregularities of articular cartilage with fibrocartilage. A $4 \mathrm{~mm}$ biopsy punch and mallet were used to harvest core specimen samples composed of full thickness cartilage attached to subchondral bone from each site selected for this study. Specimens remained covered in saline soaked gauze between each sample collection. Following OCT imaging within 24 hours of harvesting each sample was placed in a sealed container of $10 \%$ phosphate buffered formalin and refrigerated for histology.

For histology analysis, samples were rinsed and placed in a phosphate-buffered saline solution and then gross photomicrographs were made using an Olympus SZH microscope (Olympus America, Inc., Melville, NY) with an Olympus MicroFire digital imaging system (Olympus America, Inc.). Following photography, the samples were imbedded in paraffin and processed for histology. Serial sections were cut perpendicular to the tissue surface at $6 \mathrm{~mm}$ intervals, mounted on $1 \times 3$ inch glass

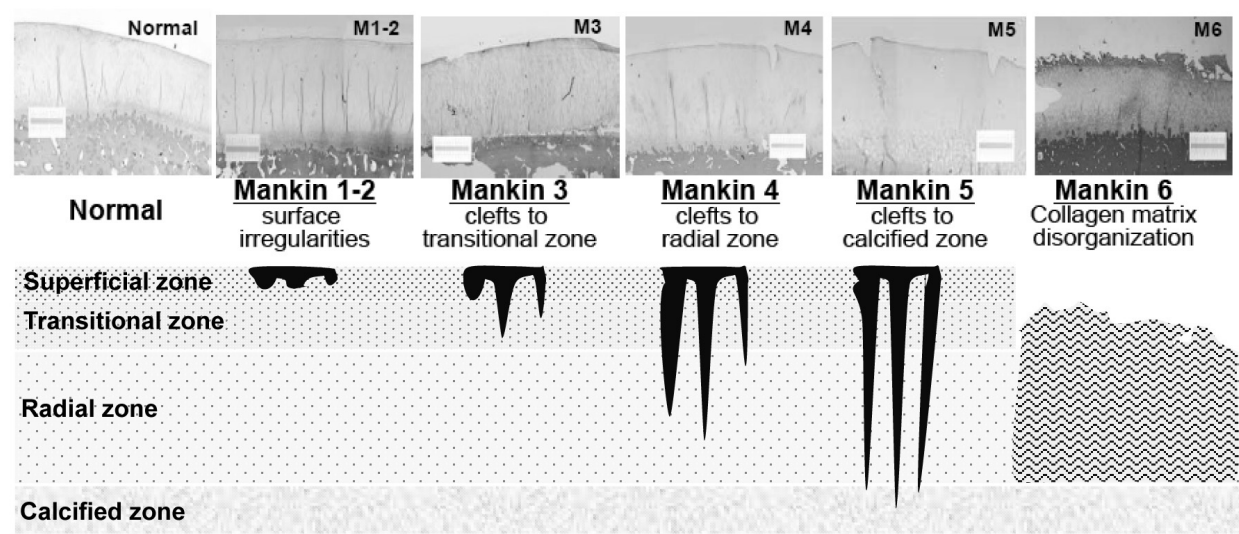

FIG. 1. Six stage histology images of degenerative cartilage classified by Mankin et al. [2] 


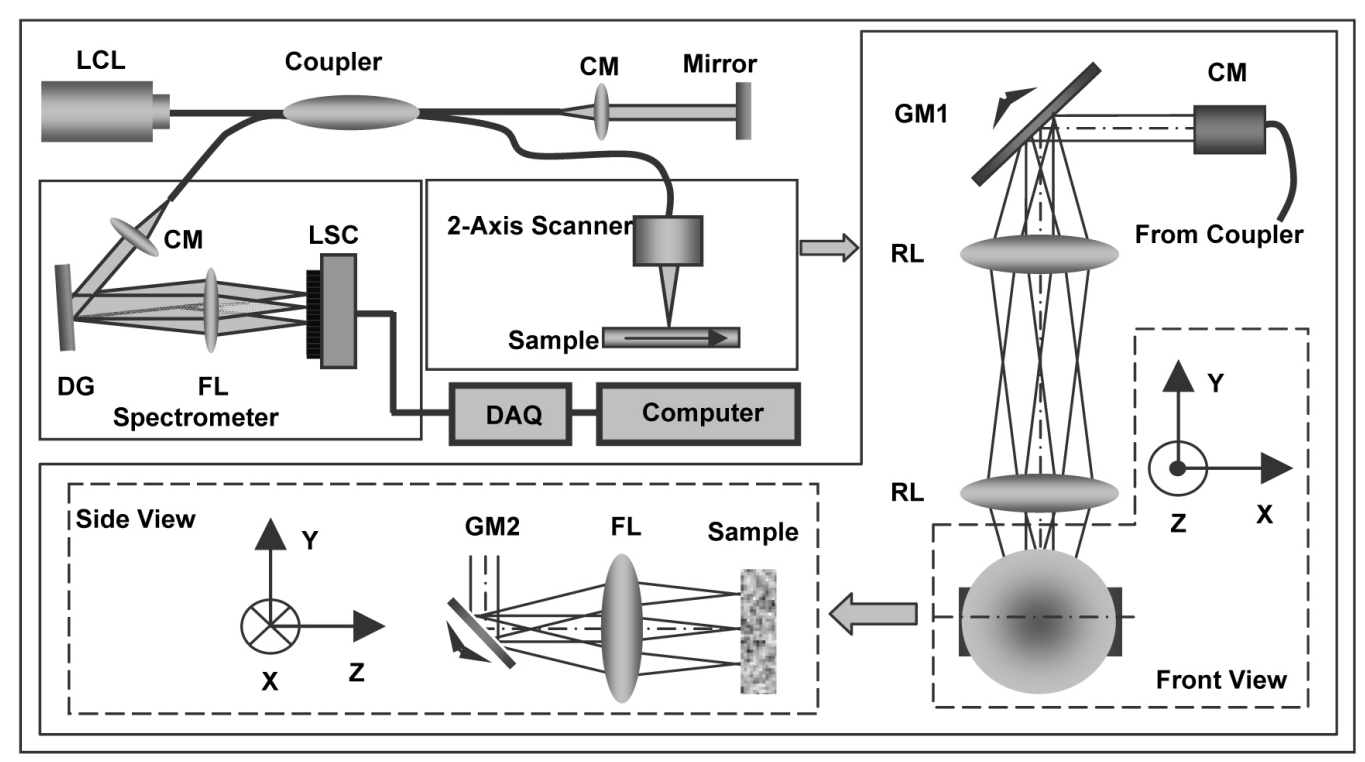

FIG. 2. Schematic diagram of the frequency domain 3-D OCT (LCL, Low-Coherence Light; CM, Collimator; DG, Diffraction Grating; FL, Focusing Lens; LSC, Line Scan Camera; GM, Galvo Mirror; RL, Relay Lens).

slides and stained with Hematoxylin and Eosin-y (H\&E).

The schematic of the frequency domain OCT is shown in Fig. 1. Low-coherence light having a center wavelength $1310 \mathrm{~nm}$ with a full width half maximum bandwidth $95 \mathrm{~nm}$ was coupled into the source arm of a fiber-based Michelson interferometer. Back-reflected lights from the reference and sample arms were guided into a spectrometer. The dispersed spectrum was sampled by a $1 \times 1024 \mathrm{InGaAs}$ detector array at $7.7 \mathrm{kHz}$. The wavelength range on the array was $130 \mathrm{~nm}$, corresponding to a spectral resolution of $0.13 \mathrm{~nm}$ and an imaging depth of $3.4 \mathrm{~mm}$ in air. In our system, a two-axis scanner with two galvanometers was used. After generating 126 individual 2-D images with $25.4 \mu \mathrm{m}$ intervals by the OCT system, we transported the images to AMIRA ${ }^{\circledR}$ software (Amira 3.1, Mercury Computer Systems Inc., Chelmsford, MA) to create 3-D volume and cross-sectional movie files. All the 2-D image data were loaded to register 3-D image datasets and generated the tissue volume of $6 \times 3.2 \times 2.5 \mathrm{~mm}^{3}$. The generated 3 -D volume images were then recorded into a movie file using a MovieMaker module that was provided by the software.

\section{RESULTS AND DISCUSSION}

Individual 2-D OCT images of the each specimen were graded according to Mankin six-stage classification that is a recognized standard for assessing degenerative cartilage. Then they were compared with histology images. As seen in the upper image of Figure 3(b), 2-D OCT image shows M-4 stage (clefts to radial zone) that is comparable with the histology image in Figure 3(c). In the same way, 2-D OCT images of all the other specimens were graded and were able to be classified by Mankin six-stage classification. Although information of individual cross-sectional images provides surface topology and structural integrity of the image site and
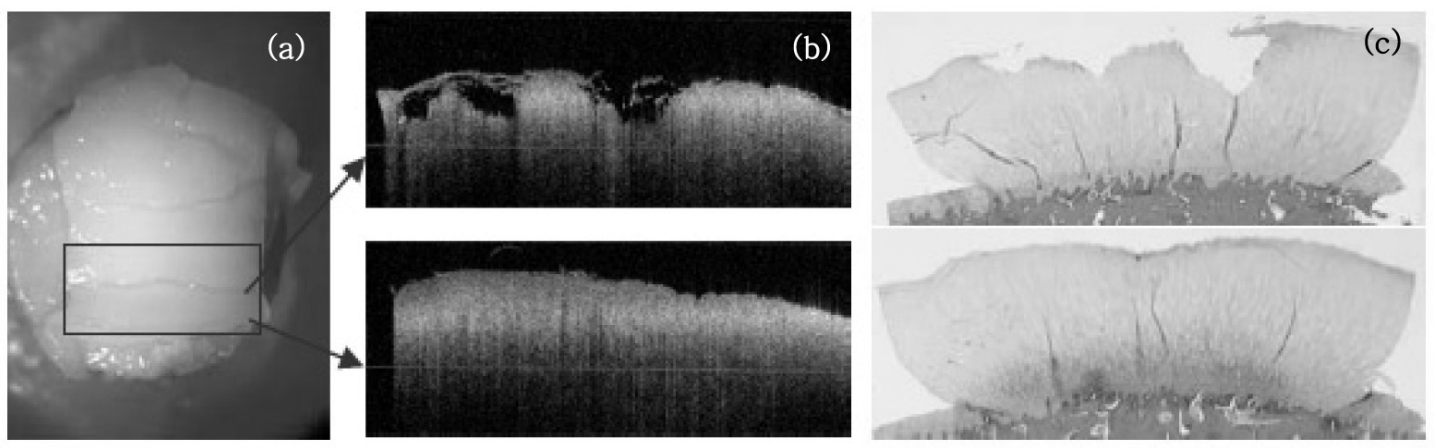

FIG. 3. Degenerative joint cartilage images: (a) Gross image extracted from a bovine knee, (b) 2-D OCT images on a cleft and normal region, and (c) matching histology images from 2-D OCT images. 

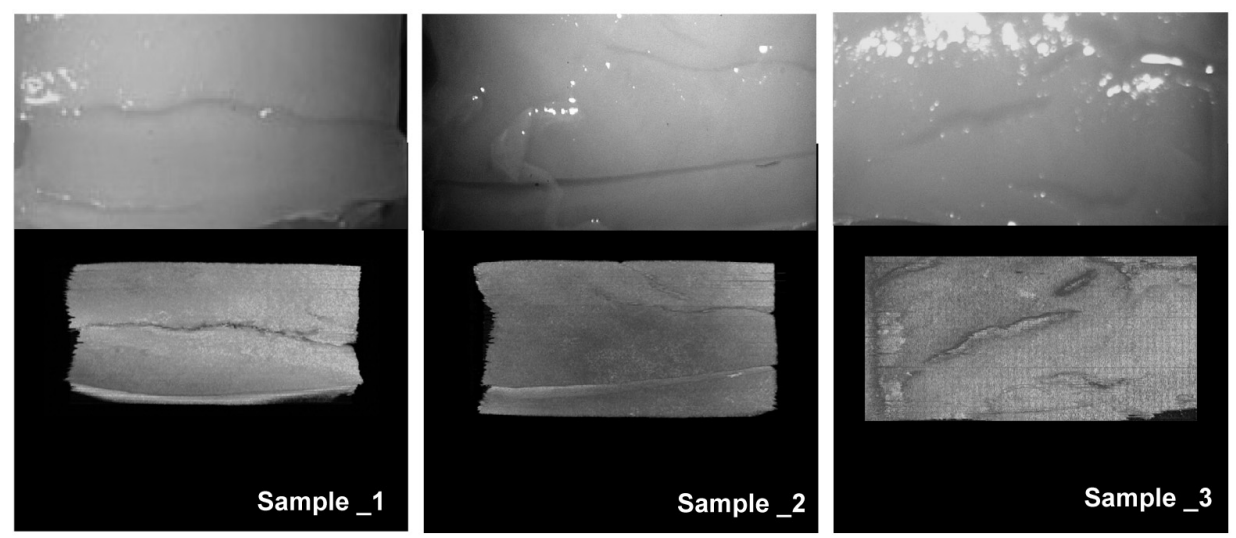

FIG. 4. 3-D Volumetric OCT images from different degenerative joint cartilage samples.

compared with histology images, it may not be fully representative of the disease within a region of a joint. Therefore, frequency domain 3-D OCT system enables a regional assessment of joint disease by allowing review of OCT images within their spatial relationship to each other as seen in Figure 4. As previously noted, volume images in Figure 4 were obtained via two-axis lateral scanner and the images were $6 \times 3.2 \times 2.5 \mathrm{~mm}^{3}$. Thresholding in the software is used to remove background in the acquired 3-D images such as air and back reflection on the sample surface, and this visualizes only biological tissue structures and morphology of interest. Figure 5 presents snapshots of 3-D OCT images of degenerative cartilage. In the volumetric images (Fig. 5(a)), tissue morphology such as clefts and surface irregularities were clearly visualized. To further evaluate the feasibility of the 3-D OCT system for potential clinical applications, cross-sectional images of the degenerative cartilage were presented to visualize the detailed cleft depth and position (Fig. 5(b)).

The results of this study indicate a high potential for clinical use of frequency domain 3-D OCT as an adjunct to conventional arthroscopy for improved diagnosis and assessment of early-stage degeneration of cartilage. The 3-D OCT image revealed surface and subsurface changes to degenerative articular cartilage that showed a high correlation with histology. Although the acquired 3-D images from all the specimens were readily graded with Mankin six-stage classification in this study, the attenuation of backscattered light and the variation of the refractive index for normal versus degenerative cartilage at a different region of cartilage may reduce the ability to image to greater depth. In these situations, the sixstage classification may not be possible due to the unclear identification between the radial zone and calcified zone within cartilage. Although the acquired images at an axial resolution of $8 \mu \mathrm{m}$ provide more detailed information than clinical MRI and ultrasound images and are comparable in structural detail to histology, this 3-D OCT system did not allow for resolution of cells. Therefore, it was not able to evaluate or assess chondrocyte cloning, viability and proteoglycan distribution that are important clues with respect to degeneration of articular cartilage. Use of improved system by using different infrared wavelengths for deep depth imaging of tissue and better resolution may permit this limi-

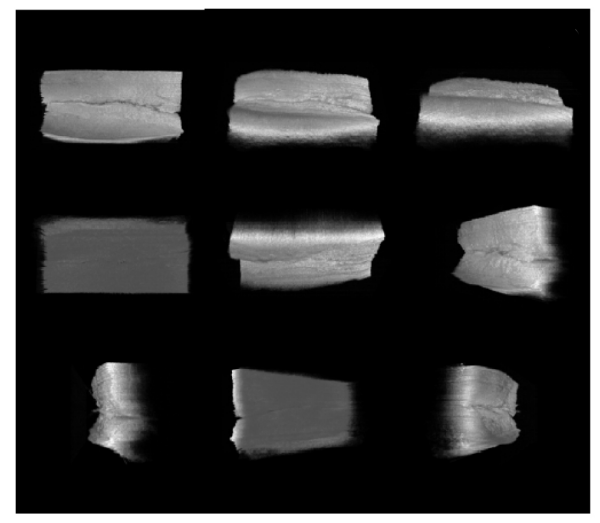

(a)

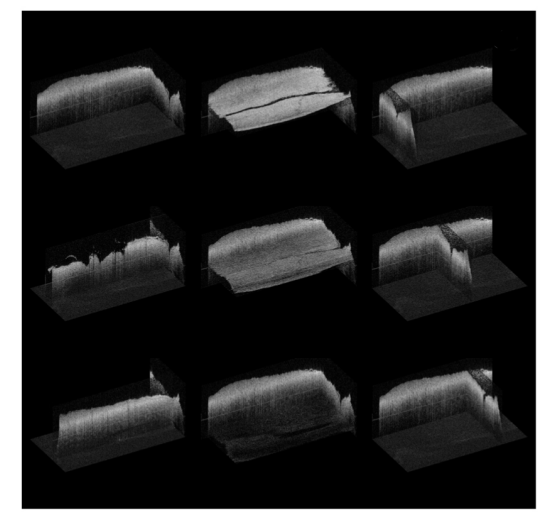

(b)

FIG. 5. Snapshots of 3-D OCT images: (a) volumetric images at different angles for the surface topography and morphology, and (b) cross-sectional images for the depth and position of a cleft. 
tation in the future. Further development of image reconstruction algorithms may also allow proteoglycan to be detected and quantified in the future. Arthroscopy integrated with this frequency domain 3-D OCT system may provide micro-structural surface topography, sub-surface feature visualization, monitoring of treatment procedure, and volumetric observation of ablation crater \& ablation efficiency evaluation as well as conventional gross images from arthroscopy.

\section{CONCLUSION}

In this study, we have realized a frequency domain 3-D OCT system employing a two-axis scanning probe and have demonstrated its potential as a real-time tool for optical biopsy in orthopedic applications. Various degenerative cartilage samples from a bovine were imaged; the resulting volume images provided morphological details such as visualization of surface roughness, clefts in the tissue surface and so on. In particular, the depth and position of clefts was readily identified in 3D images from normal region. This frequency domain 3-D OCT system adjunct to conventional arthroscopy may be clinically useful for early detection of articular cartilage degeneration in real time and nondestructive assessment of articular cartilage repair.

\section{ACKNOWLEDGEMENT}

The author appreciates Dr. George M. Peavy for his valuable advice and guidance on this study. The author also thanks Dr. Zhongping Chens's group members for their technical assistance and support on a frequency domain OCT system at Beckman Laser Institute and Medical Clinic in University of California, Irvine, USA.

\section{REFERENCES}

[1] V. C. Mow, Injury and repair of the musculoskeletal soft tissues, (American Academy of Orthopaedic Surgeons, 1988.)

[2] H. J. Mankin, H. Dorfman, L. Lippiello, and A. Zarins, "Biochemical and metabolic abnormalities in articular cartilage from osteo-arthritic human hips. II. Correlation of morphology with biochemical and metabolic data,” J. Bone Jt. Surg. Am.. vol. 53, pp. 523-537, 1971.

[3] J. M. Herrmann, C. Pitris, B. E. Bouma, S. A. Boppart, C. A. Jesser, D. L. Stamper, et al., "High resolution imaging of normal and osteoarthritic cartilage with optical coherence tomography," J. Rheumatol. vol. 26, pp. 627635, 1999.

[4] C. W. Han, C. R. Chu, N. Adachi, A. Usas, F. H. Fu, J. Huard, et al., "Analysis of rabbit articular cartilage repair after chondrocyte implantation using optical coherence tomography," Osteoarthritis and Cartilage, vol. 11, pp. 111-121, 2003.

[5] J. Rogowska, C. M. Bryant, and M. E. Brezinski, "Cartilage thickness measurements from optical coherence tomorgraphy," Opt. Soc. Am. A, vol. 20, no. 2, pp. 357367, 2003.

[6] M. J. Roberts, S. B. Adams, N. A. Patel, D. L. Stampler, M. S. Westmore, S. D. Martin, et al., "A new approach for assessing early osteoarthiritis in the rat," Anal. Bioanal. Chem., vol. 377, pp. 1003-1006, 2003.

[7] Y. Pan, Z. Li, T. Xie, and C. R. Chu, "Hand-held arthroscopic optical coherence tomography for in-vivo high-resolution imaging of articular cartilage," J. Biomed. Opt., vol. 8, no. 4, pp. 648-654, 2003.

[8] C. R. Chu, D. Lin, J. L. Geisler, C. T. Chu, F. H. Fu, and Y. Pan, "Arthroscopic microscopy of articular cartilage using optical coherence tomography," Am. Orthopaedic Society for Sports Medicine., vol. 32, no. 3, pp. 699709, 2004.

[9] X. D. Li, S. Martin, C. Pitris, R. Ghanta, D. L. Stamper, M. Harman, J. G. Fujimoto, and M. E. Brezinski, "High-resolution optical coherence tomographic imaging of osteoarthritic cartilage during open knee surgery," Arthritis Res. \& Ther., vol. 7, no. 2, pp. 318323, 2005. 